\title{
Preparation of monospecific anti-PAG antibodies for cattle pregnancy detection: use of synthetic peptides to improve specificity
}

\author{
Jimena Inés Ruiz Álvarez, Juan Manuel Teijeiro, Patricia Estela Marini*
}

Instituto de Biología Molecular y Celular de Rosario, IBR-CONICET and Facultad de Ciencias Bioquímicas y Farmacéuticas, Universidad Nacional de Rosario, Rosario, Argentina.

Email: marini@ibr.gov.ar; pmarini@fbioyf.unr.edu.ar

Received 16 February 2011; revised 7 April 2011; accepted 10 April 2011.

\begin{abstract}
Immunological methods involving pregnancy associated glycoproteins (PAG) are used for cattle pregnancy detection. The faults of these methods could be overcome by using antibodies specific for each member of the PAG family. In order to differentiate between very similar proteins, preparation of antibodies specific for peptides is a method of choice. In this work, we summarize a series of considerations regarding peptide design and choose free access NCBI, Antigenicity Plot, EMBOSS Antigenic and Expasy tools to apply them. We design peptides specific for different reported PAG members and obtain the corresponding polyclonal antibodies for five of them.
\end{abstract}

Keywords: Anti-peptide; Antibodies; Pregnancy; Cattle

\section{INTRODUCTION}

Diagnosis of pregnancy and prompt re-enlistment of "non-pregnant" cattle into an appropriate reproduction protocol are essential components of successful breeding programs. Thus, early and accurate pregnancy detection is prompted and several systems that use proteins from the pregnancy associated glycoprotein (PAG) family have been developed [1]. This family can be separated in two groups, one of which is expressed only in trophoblastic binucleated cells, being its members detectable as a group in maternal blood from the time of implantation. The concentration of this group of proteins increases steadily in plasma, peaks before parturition, and remains detectable for 2 - 3 months after calving [2]. Immunological reactions involving PAGs have been used to design commercial kits for pregnancy detection [3], for review see [4]. Variations on detection between methods are significant and may be attributed to the quality of the
anti-PAG antibodies used for each assay [4]. Anti-PAG antibodies have been developed using PAG preparations purified from placenta, which contain various PAG members, or whole PAG proteins as immunogenic material. Even when monoclonal antibodies are used, several members of the PAG family may be recognized by these antibodies, as PAGs may share the recognized epitope. Sensitivity and specificity of methods using radioimmunoassay were improved by the use of heterologous anti-PAG antibodies [3] and of monoclonal antibodies that recognize only a few members of the PAG family [5]. Persistence of some members of the PAG group pos-partum also limits the use of the currently existing methods. As different members of PAG have distinct temporal expression [2], the development of antibodies specific for them could help improve the currently existing techniques. Also, as different PAGs have distinct half lives not all the members of the family persist in pos-partum serum, thus the use of antibodies that distinguish between PAGs could permit accurate pos-partum pregnancy detection. The same would apply to the detection of wastage. The objective of this work is to develop antibodies against peptides unique to different members of the PAG family, which would presumably be specific for one reported PAG.

Anti-peptide antibodies may be obtained using recombinant proteins [6] or synthetic peptides coupled to carrier proteins [7] as antigens. The production of antibodies using synthetic peptides allows the recognition of specific regions of proteins and is often found advantageous for diagnostic purposes. The selection of the peptide sequence is essential for the immunization of the recipient organism and for the future detection of the protein or domain in its native form. In this work, free access NCBI, Antigenicity Plot, EMBOSS Antigenic and Expasy tools are used for antigenic peptide sequences selection. 


\section{METHODS}

\subsection{Peptide Design}

Uniqueness: The sequences of the 22 reported bovine PAGs were taken from the NCBI database [8] and aligned using MultAlin5.4.1. [9]. As in order to constitute an epitope a peptide must be at list 6 aminoacids (aa) long (3000 - $5000 \mathrm{Da}$ ) [7], unique sequences at least 8 aa long were chosen in this work, to assure immunogenicity. As only the 13 PAGs expressed exclusively in trophoblastic binucleated cells are candidates to be found in serum in posterior use, peptides were selected only for them (Figure 1, bold) [2]. The antibodies must recognize only the selected peptide in the context in which they will be used, thus chosen sequences need to be absent not only from other PAGs but also from other serum proteins. The BLASTp option of the NCBI database was used to search for the possible presence of the peptides in other proteins reported on the non redundant (nr) Bos taurus proteins database, which considers translated as well as determined protein sequences. This led to the elimination of 39 of the 90 considered peptides (Figure 1, underlined).

Antigenicity: The characteristics of the aa contained in a peptide determine its antigenicity in the context of the protein, where it must be recognized. From the different possible criteria, two antigenicity prediction programs were used: Antigenicity Plot [10], a tool that computes and plots the antigenicity along a polypeptide chain, as predicted by an algorithm; and EMBOSS Antigenic [11], which predicts potentially antigenic regions of a protein sequence considering experimental data. Nine peptides resulted non antigenic by any of the tools, and were not furthered analyzed.

Exposure: In order to be detected in the native protein, a peptide must be exposed in its surface. Four highly variable domains which are exposed in surface loops have been reported for PAGs [12]. Twentyseven of the considered peptides are present in these domains, and were selected for further analysis.

Posttranslational modification: If the peptide used for antibody preparation is modified in the native protein the antibodies developed against it might be unable to detect it in its context. Possible posttranslational modifications

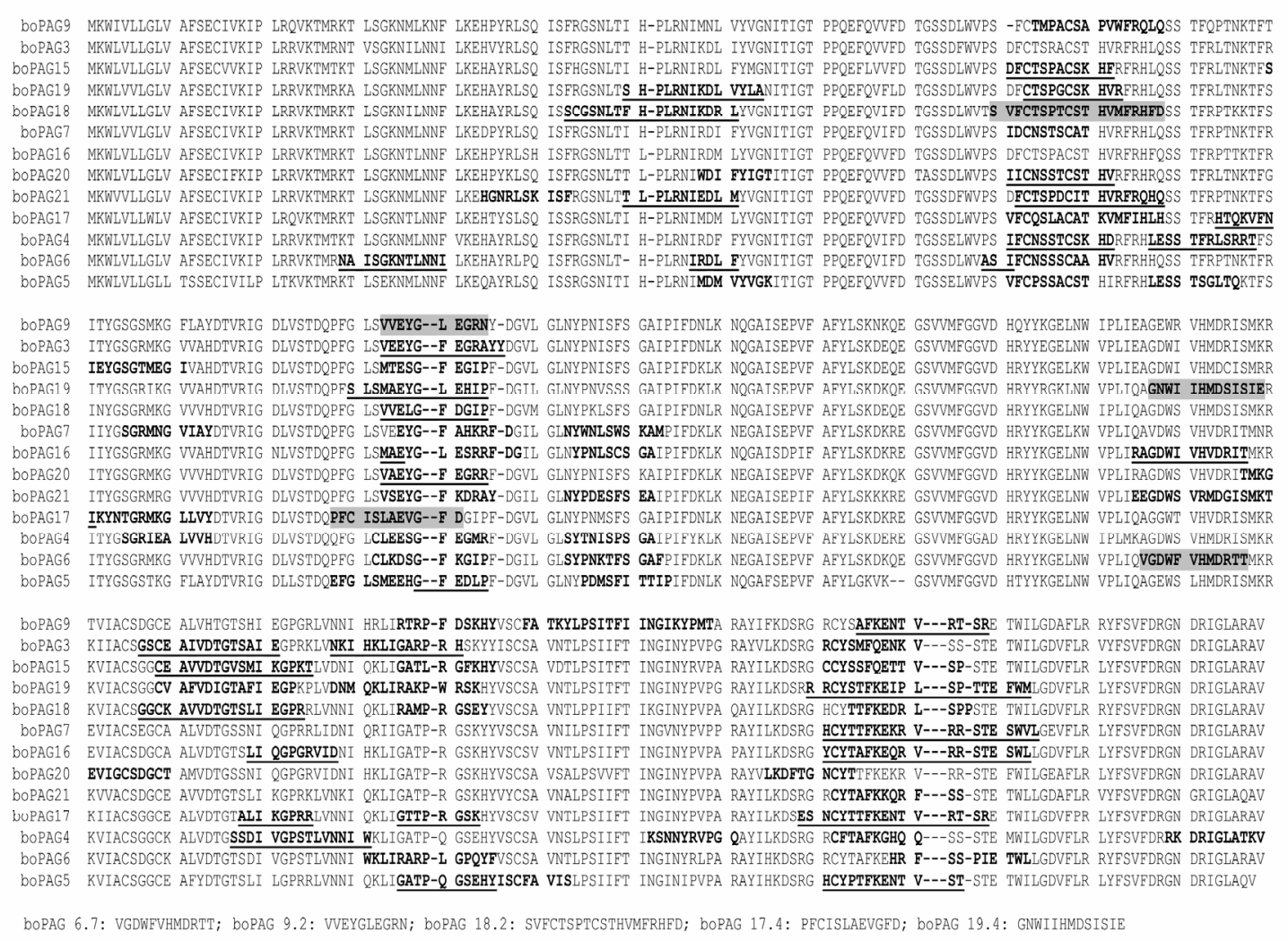

Figure 1. Alignment of the 13 bovine PAGs of probable early expression during pregnancy. Candidate peptides selected based on length and uniqueness on the PAG family are showed in bold. Peptides eliminated because of their homology to other bovine proteins are underlined. The five peptides used for antibody obtainment are highlighted and named underneath. 
and signal peptides in the protein were analyzed using the whole protein sequences with the Expasy tools [13]: NetNGlyc (N-glycosylation), YingOYang (O-betaGlcNAc attachment), NetGlycate (glycation of epsilon amino groups of lysines), NetCGlyc (C-mannosylation), NetPhos (phosphorylation), SignalP from NetNglyc (signal peptides). Posttranslational modifications were predicted for eleven candidate peptides and none of the peptides under study so far were predicted to be part of a signal sequence.

Synthesis: Difficulties in peptide synthesis compromise the purity of the product, with an augment of contaminating peptides. Peptide Calculator [14] was used to estimate peptide synthesis capability and solubility, 1 peptide was discarded.

\subsection{Antibody Production}

From the 15 peptides chosen, 5 (Figure 1) were synthezised by GenBiotech, Argentina. The peptides were coupled to rabbit serum albumin using 3-Maleimidobenzoic acid $N$-hydroxysuccinimide ester (MBS) [7]. MBS (25 mg/ml) was first linked to the peptides (5 $\mathrm{mg} / \mathrm{ml})$ and afterwards to the carrier $(10 \mathrm{mg} / \mathrm{ml})$ in order to favor linkage by the amino-terminal group of the peptide. Coupling efficiency was tested by filtrating the reaction mix through Millipore Centricon ${ }^{\circledR}$ Centrifugal Filter Units (cut-off $10 \mathrm{kDa}$ ) (Millipore, Bedford, MA, USA) followed by peptide concentration determination of the filtrate using a QuBit devise (GE Healthware). The efficiency was stated as coupled peptide (used minus filtered)/total peptide (used) and was between $80 \%$ and $85 \%$ in every case.

Four New Zealand rabbits (one rabbit per peptide) were immunized by four subcutaneous injections of 250 $\mu \mathrm{g}$ peptide with equal amounts of adjuvant. Samples were taken 30, 60 and 90 days after the first boost.

\subsection{Antibodies Quality Analysis}

The presence and peptide specificity of antibodies was analyzed by dot blot, immobilizing the peptides $(10 \mu \mathrm{g})$ and fetuin $(10 \mu \mathrm{g})$, used as specificity control for proteins in general, on PVDF membranes (Hybond-P, GE Heathware).

Placenta from 50 - 70 days pregnant cows and muscle samples were obtained at a local abattoir and transported to the laboratory on ice. Protein extracts were prepared by homogenizing cubic pieces ( $2 \mathrm{~cm}$ each side) of tissue in $5 \mathrm{ml}$ of $20 \mathrm{mM}$ Tris-HCl pH 7.6, $2 \mathrm{mM}$ EDTA, $1 \mathrm{mM}$ PMSF with Omnimixer Homogenizer (Omni International, Waterbury, CT, USA), followed by centrifugation at 27,000 $\mathrm{xg}$ for 1 hour. Protein extracts were used for native PAGE and blotted to PVDF membranes.

Antibodies were labeled with biotin using biotinylat- ing reagent EZ-Link ${ }^{\circledR}$ Sulfo-NHS-SS-Biotin according to the supplier's instructions (Pierce, Thermo Scientific, Rockford, IL, USA). PVDF membranes were blocked overnight with $1 \%$ gelatin in TTBS buffer, which consisted of $20 \mathrm{mM}$ Tris-HCl, $\mathrm{pH}$ 7.6, $150 \mathrm{mM} \mathrm{NaCl}, 0.1 \%$ v/v Tween 20, followed by two 5 min washes with TTBS. After incubation with the indicated dilution of anti-peptide antibody for $1 \mathrm{~h}$, membranes were washed twice with TTBS for $10 \mathrm{~min}$ and treated with 1:8000 dilution in TTBS of $1 \mathrm{mg} / \mathrm{ml}$ of peroxidase-conjugated streptavidin during $1 \mathrm{~h}$ at room temperature. After three washes with TTBS and one with TBS (TTBS without Tween 20), peroxidase activity was revealed using enhanced chemiluminescence detection with SuperSignal ${ }^{\mathrm{R}}$ WestPico Chemiluminiscent Substrate, Thermo Scientific, USA, according to manufacturer's instructions. Controls without anti-peptide antibodies and using pre-immunization sera were done. These showed no signal for dot blots and for western blots (data not shown).

\section{RESULTS AND DISCUSSION}

In order to obtain antibodies specific for different PAGs we aimed to establish a method to select exclusive and highly antigenic peptides for each of them, and develop the corresponding antibodies using chemically synthesized peptides. Most often, peptide exclusiveness is the only item considered for peptide design. We listed a series of considerations to be made in peptide sequence selection in order to augment the possibility for them to impress the immunological system of the animal in which the antibodies will be developed and to produce antibodies capable of recognizing the peptide in the native protein upon further use. Our simple guidelines use NCBI, Antigenicity Plot, EMBOSS Antigenic and Expasy tools, chosen from the free access available ones. To test the designed peptides we selected five of them, corresponding to different PAGs, had them synthesized, coupled them to a carrier protein and used them for antibody development in rabbits by our custom protocol, which is based on [7]. The presence of antibodies was analyzed by dot-blot, obtaining positive reaction in every case (Figure 2(a)); the corresponding controls: fetuin, assays without primary antibody and pre-immune serum, gave no signal. Dot-blot assays were done to analyze the possibility of crossed peptide detection showing each serum recognized exclusively the peptide used to produce it, indicating specificity (Figure 2(b)).

To analyze recognition of the peptides in their context, western blots of placenta native protein extracts (50 - 70 days post-insemination) were done, the results obtained using anti-PAG18 are shown (Figure 3). For anti-PAG18, two protein bands are detected in placenta that are not seen when analysis is performed with pre-immune serum 


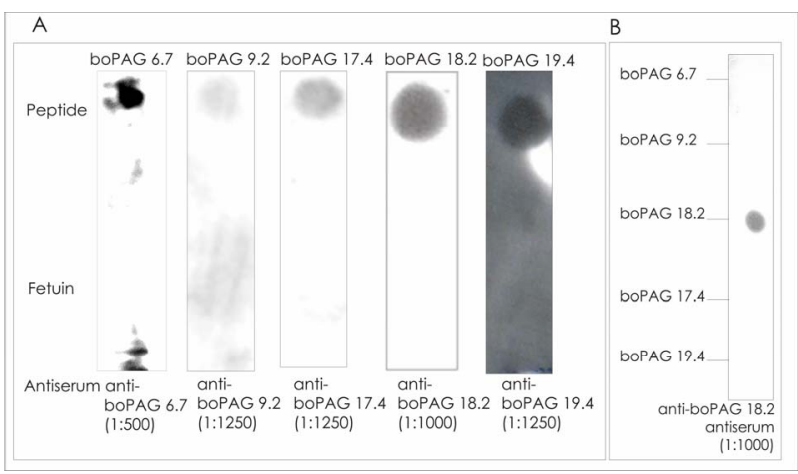

Figure 2. Specificity of anti-peptide antibodies. (a) Dot-blots of the named peptides and fetuin $(10 \mu \mathrm{g}$ each), as a negative control, in PVDF membranes with the corresponding antibodies. Dilutions are noted at the bottom; (b) A representative dot-blot of five analyzed peptides with one of the developed antibodies (anti-18.2).

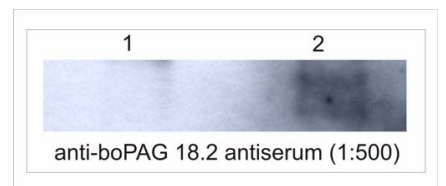

Figure 3. Recognition of peptides in the native protein. A representative result for anti-18.2 is shown. 1-Muscle and 2-Placenta protein extracts were used for non-denaturing electrophoresis followed by western blot with anti-18.2 peptide (1 : 500).

or without antibody (data not shown) or in other tissue (muscle, Figure 3, line 1). The presence of two bands may indicate different glycosylation or charge states of PAG 18, or the presence of an additional, still not reported member of the PAG family that also contains the used peptide. As more than 100 genes may encode PAGs in ruminant placenta [15] the last possibility can not be ruled out.

The considerations made for peptide design in this work allowed the development of specific antibodies in all five randomly chosen cases. The preparation of antibodies specific for different PAGs through the use of chemical peptides, designed not only based on uniqueness in PAGs but also following the other guidelines listed here, may help obtain better results in ruminant pregnancy detection. These simple guidelines may serve prepare specific antibodies for other purposes as well.

\section{ACKNOWLEDGEMENTS}

This work was supported by Secretaría de Estado de Ciencia, Tecnología e Innovación, Provincia de Santa Fe, Argentina, and Fundación Nuevo Banco de Santa Fe, Argentina.

\section{REFERENCES}

[1] Piechotta, M., Bollwein, J., Friedrich, M., Heilkenbrinker, T., Passavant, C., Branen, J., Sasser, G., Hoedemaker, M. and Bollwein, H. (2011) Comparison of commercial ELISA blood tests for early pregnancy detection in dairy cows. Journal of Reproduction and Development, 57, 72-75.

[2] Green, J.A., Xie, S., Quan, X., Bao, B., Gan, X., Mathialagan, N., Beckers, J.F. and Roberts, R.M. (2000) Pregnancy-associated bovine and ovine glycoproteins exhibit spatially and temporally distinct expression patterns during pregnancy. Biology of Reproduction, 62, 1624-1631. doi:10.1095/biolreprod62.6.1624

[3] Ayad, A., Sousa, N.M., Sulon, J., Hornick, J.L., IguerOuada, M. and Beckers, J.F. (2009) Correlation of five radioimmunoassay systems for measurement of bovine plasma pregnancy-associated glycoprotein concentrations at early pregnancy period. Research in Veterinary Science, 86, 377-382. doi:10.1016/j.rvsc.2008.10.003

[4] Whitlock, B.K. and Maxwell, H.S. (2008) Pregnancyassociated glycoproteins and pregnancy wastage in cattle. Theriogenology, 70, 550-559. doi:10.1016/j.theriogenology.2008.05.003

[5] Green, J.C., Volkmann, D.H., Poock, S.E., McGrath, M.F., Ehrhardt, M., Moseley, A.E. and Lucy, M.C. (2009) Technical note: A rapid enzyme-linked immunosorbent assay blood test for pregnancy in dairy and beef cattle. Journal of Dairy Science, 92, 3819-3824. doi:10.3168/jds.2009-2120.

[6] Saccodossi, N., De Simone, E. and Leoni, J. (2006) A novel and easy method for the production of recombinant peptides for use in the generation of monospecific antisera against Lama glama IgG2b and IgG2c subclasses. Journal of Immunological Methods, 313, 214-218. doi:10.1016/j.jim.2006.05.005

[7] Harlow, E. and Lane, D. (1988) Antibodies, a laboratory manual. Cold Spring Harbor Laboratory.

[8] NCBI database. http://www.ncbi.nlm.nih.gov/

[9] Corpet, MultAlin5.4.1. (1988) Internet available at http://bioinfo.genotoul.fr/multalin/multalin.htlm

[10] Hopp and Woods (1981) Antigenicity Plot. Internet http://www.bioinformatics.org/JaMBW/3/1/7/

[11] Kolaskar and Tongaonkar (1990) EMBOSS Antigenic. http://liv.bmc.uu.se/cgi-bin/emboss/antigenic.

[12] Hughes, A.L., Green, J.A., Garbayo, J.M. and Roberts, R.M. (2000) Adaptive diversification within a large family of recently duplicated, placentally expressed genes. Proceedings of the National Academy of Sciences USA, 97, 3319-3323. doi:10.1073/pnas.050002797

[13] Expasy tools. http://ca.expasy.org/tools/

[14] Peptide Calculator, Sigma-Aldrich. Internet available at: http://www.sigma-genosys.com/calc/pepCalc.asp

[15] Xie, S., Green, J. and Roberts, R.M. (1988) Expression of multiple genes for pregnancy-associated glycoproteins in the sheep placenta. Advances in Experimental Medicine and Biology, 436, 195-200. 